\title{
Serum 3-Hydroxybutyrate in Patients with
} Psychogenic Somatoform Symptoms May Be a Predictor of the Effectiveness of Sertraline and Venlafaxine

\author{
Norihiro Saito \\ Masamichi Itoga \\ Satoko Minakawa (DD \\ Hiroyuki Kayaba \\ Department of Clinical Laboratory \\ Medicine, Hirosaki University Graduate \\ School of Medicine, Hirosaki City, \\ Aomori, 036-8562, Japan
}

Background: Selective serotonin reuptake inhibitors (SSRIs) and serotonin-noradrenaline reuptake inhibitors (SNRIs) are often used to treat outpatients with psychogenic somatoform symptoms but prove ineffective in some cases. The metabolite 3 -hydroxybutyrate $(3 \mathrm{HB})$ is currently attracting attention as a marker of the severity of depression. We investigated whether serum $3 \mathrm{HB}$ levels in patients with psychogenic somatoform symptoms can predict the effectiveness of sertraline and venlafaxine.

Patients and Methods: Physical and psychiatric problems were assessed in 132 outpatients, and symptomatic response and serum 3HB concentrations were examined before and after treatment with sertraline $(50 \mathrm{mg} /$ day $)$ or venlafaxine $(75 \mathrm{mg} /$ day $)$.

Results: In $30.3 \%$ of patients with psychogenic symptoms, serum $3 \mathrm{HB}$ was above the upper limit of normal $(<80 \mu \mathrm{mol} / \mathrm{L})$. According to multiple logistic regression analysis, only episodes of suicidal ideation showed a significant positive association with elevated $3 \mathrm{HB}$ (odds ratio 10.2; 95\% confidence interval (CI) 2.46-42.2). The sensitivity of $3 \mathrm{HB}$ for the effectiveness of sertraline or venlafaxine for psychosomatic symptoms was $44.6 \%$, but specificity was $93.9 \%$. Hierarchical multiple logistic regression analysis identified $3 \mathrm{HB}$ as a better predictor of the effectiveness of medication (odds ratio 10.0; 95\% CI, 2.49-40.3) than episodes of suicidal ideation.

Conclusion: The present findings suggest that high serum 3HB levels in patients with psychogenic somatoform symptoms may be associated with suicidal ideation and the effectiveness of sertraline and venlafaxine at low to intermediate doses. The $3 \mathrm{HB}$ level may be a good predictor of the effectiveness of medication. Examination of serum $3 \mathrm{HB}$ levels may lead to earlier and more appropriate administration of sertraline and venlafaxine.

Keywords: 3-hydroxybutyrate, psychogenic somatoform symptoms, sertraline, venlafaxine

\section{Introduction}

Many patients with nonspecific symptoms, such as fatigue, dizziness, headache, other pain, numbness, and palpitations, consult with general practitioners. If no biological origin is found following an appropriate work-up, such symptoms may be considered psychogenic somatoform symptoms caused by mood disorders, anxiety disorders, and somatoform disorders. ${ }^{1}$ For example, over $75 \%$ of depressed patients in primary care complain of painful physical symptoms, such as headache, stomach pain, and neck and back pain, as well as non-specific generalized pain. ${ }^{2}$
Correspondence: Norihiro Saito Medicine, Hirosaki University Graduate School of Medicine, 5 Zaifu-Cho, Hirosaki City, Aomori, 036-8562, Japan

Tel +8I-0I72-33-5I II

Email norihiro@hirosaki-u.ac.jp 
Treatment begins primarily with listening, acceptance and discussion, followed by prescription of anxiolytics and antidepressants. Currently, selective serotonin reuptake inhibitors (SSRIs) and serotonin-noradrenaline reuptake inhibitors (SNRIs) are used for chronic nonspecific physical symptoms, because recent studies have shown that these drugs provide benefits for symptoms, particularly chronic pain, such as neuralgic pain, fibromyalgia, headaches, and joint pain. ${ }^{3-5}$ Physicians in Japan often prescribe SSRIs or SNRIs for prolonged nonspecific somatic symptoms of unknown origin. However, these medications are ineffective in some cases, and predicting their effectiveness is difficult.

Metabolomics has recently been utilized to develop novel biomarkers as an exploratory research tool for not only physical illness, but also mental illness. ${ }^{6}$ For example, analyses comparing concentrations among patients in remission and those with current depression revealed a pattern of metabolite alterations similar to those in analyses comparing control and currently depressed patients. One difference observed in remitted patients relative to depressed patients was an elevation in the concentration of 3-hydroxybutyrate (3HB). ${ }^{6}$ As a result, $3 \mathrm{HB}$ is currently attracting attention as a marker of the severity of depression. $^{7}$ As a type of ketone body, 3HB offers a marker of favored lipid metabolism compared to glucose metabolism and represents an energy source in the brain. Recent studies have suggested that $3 \mathrm{HB}$ is associated with brain inflammation, and may contribute to epilepsy in particular. ${ }^{8,9}$ However, few studies have assessed the clinical significance of elevated serum concentrations of $3 \mathrm{HB}$ in patients with psychogenic somatoform symptoms.

We therefore prospectively investigated the characteristics of patients with elevated serum $3 \mathrm{HB}$ levels and assessed whether $3 \mathrm{HB}$ levels in patients with psychogenic symptoms can help predict the effectiveness of sertraline and venlafaxine.

\section{Patients and Methods}

\section{Participants}

Participants were adult patients (age, 18-79 years) recruited from the Psychosomatic Unit of Kanagi Hospital from September 2015 to August 2018. Patients consulted with or were referred to the unit with psychogenic somatoform symptoms of biologically unknown origin, such as fatigue, dizziness or vertigo, headache, nonheadache pain, numbness, nausea, chest tightness, palpitations, chronic cough, and breathlessness. All patients underwent detailed examinations including blood tests, electrocardiogram, ultrasonography, and imaging studies to identify the cause of symptoms. Patients were excluded if they had any comorbid diagnoses (eg, undernutrition, diabetes, cancer, coronary artery disease, heart failure, chronic respiratory failure, dialysis) or if they were taking any psychotropic medications other than benzodiazepines. All patients were questioned regarding psychiatric and psychosomatic symptoms in addition to the general interview. Patients who reported such symptoms were interviewed using the Mini-International Neuropsychiatric Interview (MINI), ${ }^{10}$ a short structured diagnostic interview for psychiatric disorders. The level of depressive mood was scored using the Center for Epidemiologic Studies Depression Scale (CES-D), ${ }^{11}$ and the level of anxiety was measured using the State-Trait Anxiety Inventory (STAI). ${ }^{11,12}$ Psychiatric disorders were finally diagnosed according to the criteria of the Diagnostic and Statistical Manual of Mental Disorders, 5th edition (DSM-5) ${ }^{13}$ by a physician trained in psychosomatic medicine or psychiatry. Patients were excluded if they had any cognitive deficits (eg, dementia), severe psychopathology (eg, schizophrenia, delusional disorder, delirium, acute mania), or current substance abuse. Initially, 156 patients agreed to participate in this study.

\section{Measurement of $3 \mathrm{HB}$}

Levels of 3HB were measured using an enzymatic cycling assay based on an enzymatic cycling reaction in which the cofactor $\mathrm{NAD}^{+}$is reduced to NADH. NADH reacts with a colorimetric probe that produces a colored product that can be measured at $450 \mathrm{~nm}$. The intensity of the product color is proportional to the concentration of $3 \mathrm{HB}$ within a sample. ${ }^{14}$ Levels of $3 \mathrm{HB}$ were measured before treatment and at the time points described below.

\section{Treatment and Evaluation of the Effectiveness}

After laboratory testing, patients were randomly prescribed sertraline or venlafaxine. All 156 patients were initially treated for 1-2 weeks with sertraline at $25 \mathrm{mg}$ / day or venlafaxine at $37.5 \mathrm{mg} /$ day, then increased to the intermediate dose for Japanese (sertraline: $50 \mathrm{mg} /$ day; venlafaxine: $75 \mathrm{mg} /$ day) in a stepwise manner. ${ }^{15,16}$ Twenty-four patients (15.4\%) declined to continue treatment, mainly due to complaints of side effects within 2 
weeks although the medicine was at the minimum dose. They were excluded from this study. Twenty patients who complained of side effects were able to prolong treatment by switching to venlafaxine or sertraline. The final number of participants was 132. The effectiveness of treatment was determined by subjective reports from the patient of improved physical symptoms continuing $\geq 4$ weeks ("Yes or No") after 8-16 weeks of treatment. When such improvement was "Yes", $3 \mathrm{HB}$ was measured at that time. If a patient did not manifest improvement after 8 weeks and expressed a desire to end medication, 3HB was measured at that time. When patients expressed a desire to continue treatment even without improvement after 16 weeks, $3 \mathrm{HB}$ was measured in week 16 . The mean interval to $3 \mathrm{HB}$ measurement was 85.7 days (range, 56-112 days) from the beginning of treatment, with means of 77.6 days for patients with improvement and 99.4 days for patients without improvement. A total of 105 patients (79.5\%) with or without improvement continued on the same treatment for $\geq 16$ weeks depending on patient preference. The remaining 27 patients (20.5\%) without improvement desired different treatments or a referral to another hospital.

\section{Ethical Approval}

The study was conducted in accordance with the Declaration of Helsinki and approved by the ethics committee of Kanagi Hospital, and eligible patients provided written informed consent prior to enrolment.

\section{Data Analysis}

Data were analyzed using SPSS software version 23.0 (IBM, Chicago, IL, USA) and Statcel 3rd Add-in forms on Excel (OMS publication, Saitama, Japan). Two-group analysis of clinical characteristics (Tables 1 and 2) was performed using Student's $t$-test (parametric data) or the chi-square test $(2 \times 2$ contingency table data).

Associations between elevated $3 \mathrm{HB} \geq 80 \mu \mathrm{mol} / \mathrm{L}$ (reference range $<80 \mu \mathrm{mol} / \mathrm{L}$ ) and patient characteristics were investigated with multiple logistic regression analysis (Table 3). Associations between the effectiveness of sertraline or venlafaxine and patient characteristics were investigated by hierarchical multiple logistic regression analysis (Table 4). Here, the effectiveness of sertraline or venlafaxine was the dependent variable. In the first step, age, sex (female $=1$; male $=0$ ), body mass index, smoking (yes $=1$; no $=0$ ), CES-D score, scores for state and trait anxiety in the STAI, presences of physical complaints such as fatigue, dizziness (or vertigo), headache, non-headache pain, numbness, nausea, chest tightness, palpitations, chronic cough (or globus syndrome), breathlessness (yes $=1$, no $=0$ ), presence of psychological episodes, complaints such as anorexia, insomnia, diminished motivation, suicidal ideation (yes $=1$, no $=0$ ), and current use of benzodiazepines (yes $=1$, no $=0$ ) were entered as independent variables (Model 1). In the second step, elevated $3 \mathrm{HB}(\geq 80 \mu \mathrm{mol} / \mathrm{L}$; yes $=1$, no $=0$ ) was entered as an additional independent variable (Model 2). Differences in goodness of fit between adjacent models were evaluated by the change in "-2 log likelihood" values along with the change in degrees of freedom values.

In paired two-group analysis of $3 \mathrm{HB}$ measurements (Figure 1), the Wilcoxon signed-ranks test was performed because the distribution of data did not follow a normal distribution.

The significance levels of all statistical analyses were at 0.05 .

\section{Results}

In 40 of 132 patients (30.3\%) with psychogenic symptoms, serum $3 \mathrm{HB}$ was above the upper limit of normal $(\geq 80$ $\mu \mathrm{mol} / \mathrm{L}$ ) before treatment. The number and the prevalence of psychiatric disease diagnosed ultimately was 48 patients (36.4\%) with mood disorder, 21 (15.9\%) with anxiety disorder and 79 (59.8\%) with somatoform disorder (Table 1). Among the number with each disease, percentages of patients in whom treatment was non-effective were $25.0 \%$ for mood disorder, $38.1 \%$ for anxiety disorder and $45.6 \%$ for somatoform disorder.

A comparison of characteristics between the normal3HB group and high-3HB group is shown in Table 1 . Physical complaints of headache and non-headache pain were significantly more frequent in the high-3HB group $(55.5 \%, 50.0 \%)$ than in the normal-3HB group $(26.1 \%$, $27.2 \%$, respectively). Episodes of suicidal ideation occurred significantly more frequently in the high-3HB group (45.0\%) than in the normal-3HB group (8.7\%), and the number of patients whose symptoms were resolved by sertraline or venlafaxine was also significantly higher in the high-3HB group (92.5\%) than in the normal-3HB group $(50.0 \%)$.

The association between elevated 3HB and the effectiveness of treatment (sertraline or venlafaxine) was investigated. The sensitivity of high $3 \mathrm{HB}$ to the effectiveness of treatment was $44.6 \%$ and the power (detection rate) was 
Table I Comparison of Clinical Characteristics Between Normal-3HB Group and High-3HB Group

\begin{tabular}{|c|c|c|c|}
\hline & Total & Normal-3HB Group & High-3HB Group \\
\hline & $n=132$ & $\mathrm{n}=92$ & $n=40$ \\
\hline Age \pm SD & $57.2 \pm 18.6$ & $58.7 \pm 17.4$ & $53.7 \pm 21.0$ \\
\hline Female (\%) & $88(66.7 \%)$ & $62(67.4 \%)$ & $26(65.0 \%)$ \\
\hline BMI \pm SD & $23.8 \pm 3.7$ & $23.7 \pm 3.8$ & $24.1 \pm 4.3$ \\
\hline No. of smoker patients (\%) & $24(18.2 \%)$ & $16(17.4 \%)$ & $8(20.0 \%)$ \\
\hline \multicolumn{4}{|l|}{ Blood tests } \\
\hline Albumin $\pm S D(g / d L)$ & $4.21 \pm 0.33$ & $4.19 \pm 0.30$ & $4.24 \pm 0.39$ \\
\hline Creatinine $\pm \mathrm{SD}(\mathrm{mg} / \mathrm{dL})$ & $0.71 \pm 0.15$ & $0.72 \pm 0.14$ & $0.71 \pm 0.16$ \\
\hline $\mathrm{HbAlc} \pm \mathrm{SD}(\%)$ & $5.46 \pm 0.33$ & $5.48 \pm 0.34$ & $5.42 \pm 0.30$ \\
\hline \multicolumn{4}{|l|}{ Psychological tests } \\
\hline$C E S-D \pm S D$ & $22.2 \pm 13.8$ & $21.4 \pm 13.8$ & $24.1 \pm 13.8$ \\
\hline STAl-state \pm SD & $49.7 \pm 11.4$ & $49.5 \pm 12.1$ & $50.3 \pm 9.7$ \\
\hline STAl-trait \pm SD & $51.9 \pm 11.3$ & $50.9 \pm 11.5$ & $53.9 \pm 10.8$ \\
\hline Current use of benzodiazepines (\%) & $44(33.3 \%)$ & 32 (34.8\%) & $12(13.0 \%)$ \\
\hline \multicolumn{4}{|l|}{ Psychological complaints and episodes } \\
\hline Fatigue (\%) & $60(45.5 \%)$ & $39(42.4 \%)$ & $21(52.5 \%)$ \\
\hline Dizziness or vertigo (\%) & $33(25.0 \%)$ & 19 (20.7\%) & $14(35.0 \%)$ \\
\hline Headache (\%) & $46(34.8 \%)$ & $24(26.1 \%)$ & $22(55.0 \%)^{* *}$ \\
\hline Non-headache pain (\%) & $45(34.1 \%)$ & $25(27.2 \%)$ & $20(50.0 \%)^{*}$ \\
\hline Numbness (\%) & $24(18.2 \%)$ & $18(19.6 \%)$ & $6(15.0 \%)$ \\
\hline Nausea (\%) & $23(17.4 \%)$ & 17 (18.5\%) & $6(15.0 \%)$ \\
\hline Chest tightness (\%) & $24(18.2 \%)$ & $18(19.6 \%)$ & $6(15.0 \%)$ \\
\hline Palpitation (\%) & $26(19.7 \%)$ & $18(19.6 \%)$ & $8(20.0 \%)$ \\
\hline Chronic cough or globus syndrome (\%) & $28(21.2 \%)$ & $20(21.7 \%)$ & $8(20.0 \%)$ \\
\hline Breathlessness (\%) & $8(6.1 \%)$ & $6(6.5 \%)$ & $2(5.0 \%)$ \\
\hline \multicolumn{4}{|l|}{ Psychological complaints and episodes } \\
\hline Anorexia (\%) & $36(27.3 \%)$ & $22(23.9 \%)$ & $14(35.0 \%)$ \\
\hline Insominia (\%) & 87 (65.9\%) & $63(68.5 \%)$ & $24(60.0 \%)$ \\
\hline Diminished motivation (\%) & 85 (64.4\%) & $57(62.0 \%)$ & $28(70.0 \%)$ \\
\hline Suicidal ideation (\%) & $26(19.7 \%)$ & $8(8.7 \%)$ & $18(45.0 \%)^{* * *}$ \\
\hline \multicolumn{4}{|l|}{ Psychiatric diagnosis" (ultimately) } \\
\hline Mood disorder (\%) & $48(36.4 \%)$ & $32(34.8 \%)$ & $16(40.0 \%)$ \\
\hline$<$ Major depression (\%)> & $29(22.0 \%)$ & $18(19.6 \%)$ & II (27.5\%) \\
\hline Anxiety disorder (\%) & $2 \mathrm{l}(15.9 \%)$ & $16(17.4 \%)$ & $5(12.5 \%)$ \\
\hline Somatoform disorder (\%) & 79 (59.8\%) & $54(58.7 \%)$ & $25(62.5 \%)$ \\
\hline Treatment-effective patients (\%) & $83(62.9 \%)$ & $46(50.0 \%)$ & $37(92.5 \%) * * *$ \\
\hline
\end{tabular}

Note: *Significance compared with normal-3HB group $\left({ }^{*} \mathrm{p}<0.05,{ }^{*} \mathrm{p}<0.01,{ }^{* *} \mathrm{p}<0.00 \mathrm{I}\right) .{ }^{*}$ Diagnosis is overlapped in some patients.

0.45 , but specificity was $93.9 \%$, the positive likelihood ratio was 7.28 and the positive predictive value was $92.5 \%$.

Multiple logistic regression analysis for predicting 3HB elevation was performed (Table 3). The predictive accuracy of the regression model was $80.3 \%$. In this analysis, only episodes of suicidal ideation showed a significant positive association with elevated 3HB. The odds ratio (OR) was 10.2 (95\% confidence interval (CI), 2.46-42.2).

Next, we classified the 132 patients into those whose symptoms were not resolved (non-effective group, $\mathrm{n}=49$, 
Table 2 Comparison of Characteristics Between Treatment Non-Effective Group and Effective Group

\begin{tabular}{|c|c|c|}
\hline \multirow[t]{2}{*}{$n=132$} & Non-Effective Group & Effective Group \\
\hline & $n=49$ & $\mathrm{n}=\mathbf{8 3}$ \\
\hline Age \pm SD & $58.5 \pm 19.1$ & $56.4 \pm 18.4$ \\
\hline Female (\%) & $34(69.4 \%)$ & $54(65.1 \%)$ \\
\hline BMI \pm SD & $24.2 \pm 3.8$ & $23.6 \pm 3.6$ \\
\hline No. of smoker patients (\%) & $6(12.2 \%)$ & $18(21.7 \%)$ \\
\hline \multicolumn{3}{|l|}{ Blood tests } \\
\hline Albumin $\pm S D(g / d L)$ & $4.26 \pm 0.33$ & $4.18 \pm 0.33$ \\
\hline Creatinine $\pm \mathrm{SD}(\mathrm{mg} / \mathrm{dL})$ & $0.71 \pm 0.12$ & $0.71 \pm 0.16$ \\
\hline $\mathrm{HbAlc} \pm \mathrm{SD}(\%)$ & $5.47 \pm 0.30$ & $5.46 \pm 0.34$ \\
\hline $3 \mathrm{HB} \pm \mathrm{SD}(\mu \mathrm{mol} / \mathrm{L})$ & $49.0 \pm 46.8$ & $81.0 \pm 62.0 * * *$ \\
\hline before treatment $(\%)$ & & \\
\hline $\begin{array}{l}\text { No. of patients with high-3HB }{ }^{\#} \text { before treatment } \\
\text { (\%) }\end{array}$ & $3(6.1 \%)$ & $37(44.6 \%) * * *$ \\
\hline \multicolumn{3}{|l|}{ Psychological tests } \\
\hline$C E S-D \pm S D$ & $19.6 \pm 12.4$ & $23.7 \pm 14.4$ \\
\hline STAl-state \pm SD & $48.8 \pm 12.9$ & $50.3 \pm 10.5$ \\
\hline STAI-trait \pm SD & $50.8 \pm 11.5$ & $52.5 \pm 11.3$ \\
\hline Current use of benzodiazepines (\%) & $17(34.7 \%)$ & $27(32.5 \%)$ \\
\hline \multicolumn{3}{|l|}{ Physical complaints and episodes } \\
\hline Fatigue (\%) & 18 (36.7\%) & $42(50.6 \%)$ \\
\hline Dizziness or vertigo (\%) & $12(24.5 \%)$ & $21(25.3 \%)$ \\
\hline Headache (\%) & II (22.5\%) & $34(41.1 \%)^{*}$ \\
\hline Non-headache pain (\%) & $12(25.0 \%)$ & $33(40.1 \%)$ \\
\hline Numbness (\%) & II (22.5\%) & $13(15.7 \%)$ \\
\hline Nausea (\%) & $9(18.4 \%)$ & $14(16.9 \%)$ \\
\hline Chest tightness (\%) & II (22.5\%) & $13(15.7 \%)$ \\
\hline Palpitation (\%) & II (22.5\%) & $15(18.1 \%)$ \\
\hline Chronic cough or globus syndrome (\%) & $12(25.0 \%)$ & $16(19.3 \%)$ \\
\hline Breathlessness (\%) & $3(6.1 \%)$ & $5(6.0 \%)$ \\
\hline \multicolumn{3}{|l|}{ Psychological complaints and episodes } \\
\hline Anorexia (\%) & $13(26.5 \%)$ & $23(27.7 \%)$ \\
\hline Insominia (\%) & $33(67.4 \%)$ & $54(65.1 \%)$ \\
\hline Diminished motivation (\%) & $28(57.1 \%)$ & $57(68.7 \%)$ \\
\hline Suicidal ideation (\%) & $2(4.1 \%)$ & $24(29.0 \%) * * *$ \\
\hline \multicolumn{3}{|l|}{ The ultimate psychiatric diagnosis $\$$} \\
\hline Mood disorder (\%) & $12(24.5 \%)$ & $36(43.4 \%) *$ \\
\hline$<$ Major depression (\%)> & 7 (I5.3\%) & $23(27.7 \%)$ \\
\hline Anxiety disorder (\%) & $8(16.3 \%)$ & $13(15.7 \%)$ \\
\hline Somatoform disorder (\%) & $36(73.5 \%)$ & 43 (5I.8\%)* \\
\hline
\end{tabular}

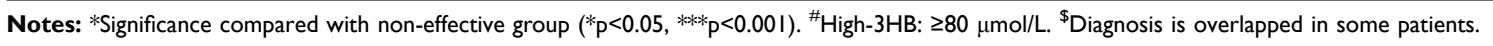

$37.1 \%)$ and those whose symptoms were resolved by sertraline or venlafaxine (effective group, $\mathrm{n}=83$, $62.9 \%)$. Characteristics were then compared between the two groups (Table 2). The level of $3 \mathrm{HB}$ before treatment was significantly higher in the effective group (mean \pm standard deviation, $81.0 \pm 62.0 \mu \mathrm{mol} / \mathrm{L})$ than in the noneffective group $(49.0 \pm 46.8 \mu \mathrm{mol} / \mathrm{L})$. The number of patients with high-3HB $(\geq 80 \mu \mathrm{mol} / \mathrm{L})$ before treatment was also significantly more frequent in the effective group $(\mathrm{n}=37,44.6 \%)$ than in the non-effective group ( $\mathrm{n}$ 
Table 3 Multiple Logistic Regression Analysis of the Factors Associated with Elevation of $3 \mathrm{HB}$

\begin{tabular}{|c|c|c|}
\hline \multirow{2}{*}{$\begin{array}{l}\mathrm{n}=132 \\
\text { Independent Valuables }\end{array}$} & \multicolumn{2}{|c|}{ Regression Model } \\
\hline & Odds Ratio & $\mathbf{p}$ \\
\hline Age & $0.99(0.97-1.02)$ & 0.627 \\
\hline Gender $($ Female $=I$, Male $=0)$ & $1.18(0.42-3.34)$ & 0.753 \\
\hline BMI & $1.06(0.93-1.21)$ & 0.397 \\
\hline Smoking & $0.89(0.26-3.10)$ & 0.859 \\
\hline CES-D & $0.99(0.93-1.04)$ & 0.615 \\
\hline STAI-state & $0.95(0.88-1.02)$ & 0.144 \\
\hline STAI-trait & $1.04(0.97-1.12)$ & 0.257 \\
\hline Current use of benzodiazepines & $0.7 \mid(0.23-2.23)$ & 0.558 \\
\hline Fatigue & $1.32(0.45-3.87)$ & 0.619 \\
\hline Dizziness or vertigo & $1.03(0.29-3.57)$ & 0.967 \\
\hline Headache & $2.64(0.80-8.72)$ & 0.112 \\
\hline Non-headache pain & I.83 $(0.52-6.37)$ & 0.345 \\
\hline Numbness & $0.55(0.13-2.38)$ & 0.426 \\
\hline Nausea & $0.49(0.12-2.04)$ & 0.324 \\
\hline Chest tightness & $0.87(0.22-3.4 I)$ & 0.847 \\
\hline Palpitation & $1.91(0.50-7.33)$ & 0.347 \\
\hline Chronic cough or globus syndrome & $1.85(0.52-6.63)$ & 0.346 \\
\hline Breathlessness & $0.77(0.09-6.88)$ & 0.814 \\
\hline Anorexia & $1.97(0.63-6.14)$ & 0.240 \\
\hline Insomnia & $0.40(0.1 \mathrm{I}-\mathrm{I} .4 \mathrm{I})$ & 0.154 \\
\hline Diminished motivation & $1.45(0.42-4.94)$ & 0.556 \\
\hline Suicidal ideation & $10.2(2.46-42.2)$ & $0.001 * *$ \\
\hline Predictive accuracy (\%) & \multicolumn{2}{|l|}{80.3} \\
\hline-2 Log likelihood & \multicolumn{2}{|l|}{121.8} \\
\hline
\end{tabular}

Note: $95 \%$ confidence intervals are included in parentheses. ${ }^{* *} p<0.01$.

$=3,6.1 \%)$. Headache and suicidal ideation were significantly more frequent in the effective group $(41.0 \%$ and $28.9 \%$, respectively) than in the non-effective group ( $22.4 \%$ and $4.1 \%$, respectively). Regarding the ultimate psychiatric diagnosis, mood disorder was significantly more frequent (43.4\%) and somatoform disorder was less frequent $(51.8 \%)$ in the effective group than in the noneffective group ( $24.5 \%$ and $73.5 \%$, respectively).

Hierarchical multiple logistic regression analysis predicting the effectiveness of medication was performed (Table 4). Regression Model 1, which excluded the factor of high 3HB, showed that an episode of suicidal ideation had a significant positive association with the effectiveness of sertraline or venlafaxine $(\mathrm{OR}=6.91,95 \%$ CI 1.31-36.4). Regression Model 2, which included high 3HB, showed that only high $3 \mathrm{HB}$ had a significant positive association with the effectiveness of medication ( $\mathrm{OR}=10.0,95 \%$ CI $2.49-40.3$ ). The predictive accuracy of regression Model 1 was $72.0 \%$ and that of Model 2 was $74.2 \%$. The $p$ value for the $\Delta-2 \log$ likelihood change was less than 0.001 , meaning that Model 2 was significantly more accurate than Model 1 .

Similarly, hierarchical multiple logistic regression analysis was performed for sertraline $(\mathrm{n}=72)$ and venlafaxine $(\mathrm{n}=60)$. In patients treated with sertraline, Model 1, which excluded $3 \mathrm{HB}$, showed that suicidal ideation had a significant positive association with effectiveness $(\mathrm{OR}=19.6,95 \%$ CI 1.47-261.4), and Model 2, which included 3HB, showed that only high $3 \mathrm{HB}$ had a significant positive association with the effectiveness of sertraline (OR $=19.9,95 \%$ CI 1.87-211.2). The predictive accuracy of Model 1 was $69.4 \%$, and that of Model 2 was $72.2 \%$. The $\mathrm{p}$ value for the $\Delta-2 \log$ likelihood change was 0.007 . In patients treated with venlafaxine, Model 1 showed that no factor was significantly associated with effectiveness, and Model 2, which included 3HB, showed that only high $3 \mathrm{HB}$ had a significant positive association with effectiveness (OR $=21.1,95 \%$ CI 1.14-391.3). The predictive accuracy of Model 1 was $73.3 \%$, and that of Model 2 was $76.7 \%$. The $\mathrm{p}$ value for the $\Delta-2 \log$ likelihood change was 0.011 .

Levels of $3 \mathrm{HB}$ before and after treatment in the noneffective group $(n=49)$ and effective group $(n=83)$ are shown in Figure 1. The 3HB level was significantly decreased in the effective group $(\mathrm{p}<0.001)$.

\section{Discussion}

General practitioners see many patients who complain of psychogenic somatoform symptoms and who may or may not have a psychiatric diagnosis. Interestingly, approximately $30 \%$ of patients with psychogenic somatoform symptoms showed elevated serum 3HB. No prospective study has evaluated the efficacy of assaying $3 \mathrm{HB}$ except in diabetic ketoacidosis. ${ }^{17-19}$

As an endogenous ketone body, 3HB supports mammalian cell metabolism during states of energy deficiency, such as those induced by fasting or exercise. ${ }^{20,21}$ In recent years, several reports have shown that $3 \mathrm{HB}$ may have antidepressant effects, ${ }^{22,23}$ likely by inhibiting the activation of the nucleotide-binding domain, leucine-rich repeat and pyrin domain-containing 3 (NLRP3) inflammasome. ${ }^{9}$

We therefore hypothesized that elevated 3HB may correlate with a diagnosis of mood disorder (especially depression) or the CES-D score; but found no significant correlation. Furthermore, a comparison of $3 \mathrm{HB}$ among mood disorders, anxiety disorders, and somatoform 
Table 4 Hierarchical Multiple Logistic Regression Analysis of the Factors Associated with Effectiveness of Sertraline or Venlafaxine

\begin{tabular}{|c|c|c|c|c|}
\hline \multirow{2}{*}{$\begin{array}{l}\mathrm{n}=132 \\
\text { Independent Valuables }\end{array}$} & \multicolumn{2}{|l|}{ Model I } & \multicolumn{2}{|l|}{ Model 2} \\
\hline & Odds Ratio & $\mathbf{p}$ & Odds Ratio & $\mathbf{p}$ \\
\hline Age & I.0I (0.98-I.03) & 0.548 & I.0I (0.99-I.04) & 0.337 \\
\hline Gender $($ Female $=I$, Male $=0)$ & $0.98(0.38-2.5 \mathrm{I})$ & 0.969 & $1.02(0.37-2.80)$ & 0.977 \\
\hline BMI & $0.96(0.85-1.08)$ & 0.465 & $0.94(0.83-1.07)$ & 0.361 \\
\hline Smoking & $1.76(0.57-5.45)$ & 0.325 & $1.98(0.60-6.5 \mathrm{I})$ & 0.261 \\
\hline CES-D & $1.02(0.97-1.07)$ & 0.404 & $1.02(0.97-1.07)$ & 0.366 \\
\hline STAI-state & $1.00(0.94-1.06)$ & 0.931 & $1.02(0.95-1.08)$ & 0.610 \\
\hline STAl-trait & $0.98(0.92-1.04)$ & 0.535 & $0.96(0.90-1.03)$ & $0.25 \mathrm{I}$ \\
\hline Current use of benzodiazepines & $0.86(0.33-2.20)$ & 0.749 & $0.93(0.34-2.55)$ & 0.886 \\
\hline Fatigue & $1.81(0.66-4.97)$ & 0.253 & $2.01(0.66-6.16)$ & 0.221 \\
\hline Dizziness or vertigo & $0.66(0.21-2.06)$ & 0.471 & $0.67(0.20-2.25)$ & 0.514 \\
\hline Headache & $1.73(0.57-5.25)$ & 0.335 & $\mathrm{I} .40(0.43-4.57)$ & 0.582 \\
\hline Non-headache pain & $1.86(0.56-6.12)$ & 0.310 & $\mathrm{I} .64(0.46-5.83)$ & 0.445 \\
\hline Numbness & $0.44(0.13-1.55)$ & 0.203 & $0.51(0.13-2.05)$ & 0.346 \\
\hline Nausea & $0.64(0.19-2.09)$ & 0.457 & $0.79(0.22-2.78)$ & 0.709 \\
\hline Chest tightness & $0.67(0.22-2.07)$ & 0.489 & $0.7 \mathrm{I}(0.2 \mathrm{I}-2.37)$ & 0.574 \\
\hline Palpitation & $1.19(0.39-3.69)$ & 0.758 & $\mathrm{I} .03(0.30-3.5 \mathrm{I})$ & 0.962 \\
\hline Chronic cough or globus syndrome & $1.02(0.32-3.28)$ & 0.976 & $0.97(0.25-3.80)$ & 0.967 \\
\hline Breathlessness & $1.40(0.24-8.23)$ & 0.710 & $1.64(0.24-1 \mathrm{I} .2)$ & 0.616 \\
\hline Anorexia & $0.78(0.28-2.14)$ & 0.628 & $0.60(0.20-1.79)$ & 0.357 \\
\hline Insomnia & $0.68(0.24-1.95)$ & 0.473 & $0.86(0.26-2.81)$ & 0.801 \\
\hline Diminished motivation & $1.02(0.37-2.85)$ & 0.965 & $0.88(0.28-2.75)$ & 0.822 \\
\hline Suicidal ideation & $6.91(1.31-36.4)$ & $0.022 *$ & $3.30(0.54-20.2)$ & 0.197 \\
\hline High-3HB $(\geq 80 \mu \mathrm{mol} / \mathrm{L})$ & & & $10.0(2.49-40.3)$ & $0.001 * *$ \\
\hline Predictive accuracy (\%) & \multicolumn{2}{|c|}{72.0} & \multicolumn{2}{|c|}{74.2} \\
\hline-2 Log likelihood & \multicolumn{2}{|c|}{151.8} & \multicolumn{2}{|c|}{134.7} \\
\hline p value for $\Delta-2$ Log likelihood change & \multicolumn{4}{|c|}{$<0.001 * * *$} \\
\hline
\end{tabular}

Notes: $95 \%$ confidence intervals are included in parentheses. ${ }^{*} p<0.05,{ }^{* *} p<0.01,{ }^{* * *} p<0.001$.

disorders revealed no differences (data not shown). These results suggest that elevated $3 \mathrm{HB}$ is not specific to depression, but rather may reflect psychosomatic stress.

Initially, patients were classified into those with normal $3 \mathrm{HB}$ and high $3 \mathrm{HB}$ in our study. Headache, non-headache pain, and suicidal ideation were more frequently found in patients in the high-3HB group. However, multiple logistic regression analysis showed that only the factor of suicidal ideation was associated with elevated 3HB. Setoyama et al have reported that $3 \mathrm{HB}$ is associated with suicidal ideation and that $3 \mathrm{HB}$ itself may control emotional systems in the brain via energy metabolite processes. ${ }^{7}$ Our results support this notion in not only patients with depression, but also patients with psychogenic somatoform symptoms. Additional studies are needed to clarify the types of patient in whom $3 \mathrm{HB}$ is elevated and the mechanisms of elevation for $3 \mathrm{HB}$.
We found frequent improvement in symptoms among high-3HB patients, and therefore explored the correlation between elevated $3 \mathrm{HB}$ and the effectiveness of sertraline or venlafaxine by classifying patients into a non-effective group and an effective group. The sensitivity of high $3 \mathrm{HB}$ to the effectiveness of pharmacotherapy was $44.6 \%$, but the specificity was $93.9 \%$, suggesting that sertraline and venlafaxine may be appropriate for patients with high $3 \mathrm{HB}$, although the effectiveness of medication cannot be negated even if $3 \mathrm{HB}$ is below the upper limit. Analysis of non-effective and effective groups also showed that levels of $3 \mathrm{HB}$ were higher in the effective group and that the number of high-3HB patients with suicidal ideation was higher than that in the non-effective group. Hierarchical multiple logistic regression analysis detected two factors, suicidal ideation and high $3 \mathrm{HB}$, and showed that $3 \mathrm{HB}$ was 

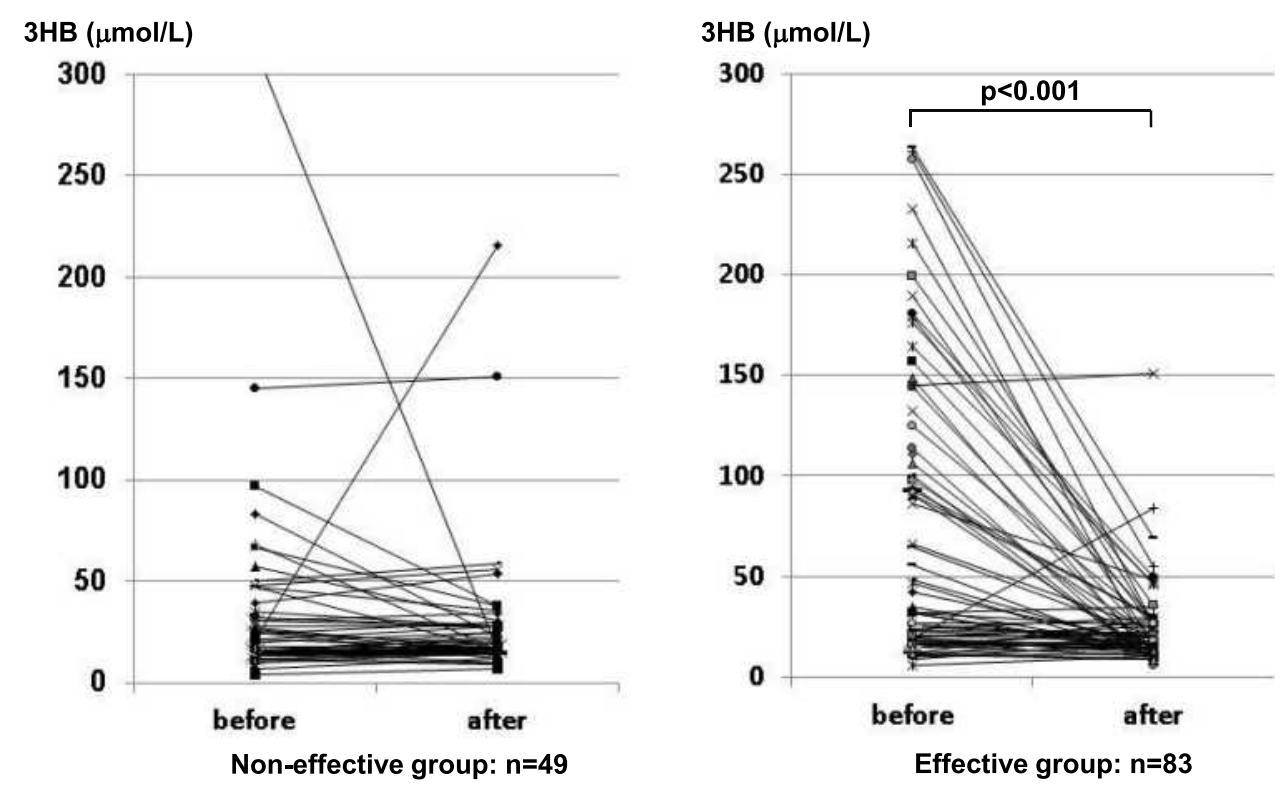

Figure I Levels of 3HB before and after treatment with sertraline or venlafaxine in the non-effective group and effective group.

a better predictor of the effectiveness of medication than episodes of suicidal ideation. These results imply that patients in whom $3 \mathrm{HB}$ was susceptible to being produced physiologically against psychosomatic stress tend to benefit from treatment with sertraline and venlafaxine, and the elevated $3 \mathrm{HB}$ was greatly decreased with the improvement of symptoms (Figure 1). NLRP3-mediated inflammation may be inhibited by $3 \mathrm{HB}$ even in patients with psychosomatic disorders other than depression. Yamanishi et al reported that peripheral administration of $3 \mathrm{HB}$ decreased inflammatory cytokine concentrations in the hippocampus, such as IL-1 $\beta$ and TNF- $\alpha$, and improved depressive and anxiety-like behaviors in a rodent model of chronic unpredictable stress. ${ }^{23}$ This suggests that $3 \mathrm{HB}$ may be a novel therapeutic candidate for the treatment of stress-related mood disorders. ${ }^{23,24}$

In addition to episodes of suicidal ideation as a common symptom of depression, headache was more frequent not only in 3HB-elevated patients (Table 1), but also in patients in whom sertraline or venlafaxine was effective (Table 2). On the other hand, CES-D score (before treatment) and prevalence of major depression were unrelated to 3HB level (Table 1), and neither related to the effectiveness of treatment with low-to-intermediate dose of sertraline or venlafaxine (Table 2) in samples of our study focused on somatic symptoms. One study reported that the SSRI fluvoxamine was beneficial for the treatment of chronic tension-type headache in not only depressed patients, but also non-depressed patients, ${ }^{25}$ and two studies have reported a significant effect of sertraline for noncardiac chest pain without any significant change in mood between a sertraline group and a placebo group. ${ }^{26,27}$ Such findings suggest that the effect of SSRIs on pain outcomes is not associated with improvements in mood.

Furthermore, the length of evaluation from the beginning of treatment (Patients and methods), meaning close to the period in which sufficient relief from symptoms was obtained for at least 4 weeks, was 77.6 days on average, compared to 99.4 days in patients without improvement (inevitably showing a significant difference), which suggests that about 7-11 weeks is needed to come to realize improvement of somatic symptoms. Aikens et al reported that in patients with depression, improvement in somatic complaints, such as headache, low back pain and stomach pain plateaued earlier than improvement in depressive symptoms and the improvement of somatic complaints was concentrated within the first month of therapy before essentially leveling off. ${ }^{28}$ This discrepancy in time may be attributed to samples focused on somatic symptoms and treatment with the sparing dose of medicine used in our study.

Collectively, our results demonstrated that the level of 3HB was initially elevated (before treatment) and decreased after treatment with low-to-intermediate doses of sertraline or venlafaxine in most patients who showed improvement of symptoms. This suggests that if $3 \mathrm{HB}$ is high in patients with psychogenic somatoform symptoms, 
3HB may offer a promising predictive biomarker for the effectiveness of sertraline and venlafaxine at low-tointermediate doses.

\section{Study Limitations}

A number of limitations should be considered when interpreting our findings. First, because this study was carried out without a placebo control, the possibility must be considered that the symptoms of patients improved due to factors other than sertraline or venlafaxine. For example, regular listening, acceptance, and discussion are very important for patients with nonspecific symptoms and may provide sufficient treatment in some cases. Physiologically elevated $3 \mathrm{HB}$ may be a harbinger of improvements in neurological stress.

Second, our study was limited regarding the doses of sertraline and venlafaxine. The Japanese drug information sheets for patients for sertraline and venlafaxine from Pfizer Pharmaceuticals (Tokyo, Japan) ${ }^{15,16}$ state.

In general, start with taking $25 \mathrm{mg}$ of sertraline once a day, and gradually increase the dosage up to $100 \mathrm{mg}$ /day

and

In general, start with taking $37.5 \mathrm{mg}$ of venlafaxine once a day. After a week, take $75 \mathrm{mg}$ once a day. The dosage may be adjusted up to $225 \mathrm{mg}$ /day according to the age or symptoms.

Thus, all patients were initially treated with a settled low-dose medication and increased to the intermediate dose for Japanese (sertraline: $50 \mathrm{mg} /$ day; venlafaxine: $75 \mathrm{mg} /$ day) and the dosage was continued to the end of this study. Those doses of both drugs are generally considered insufficient for the treatment of depression, ${ }^{29,30}$ especially for venlafaxine, which at a dose of $75 \mathrm{mg} /$ day only exerts effects as an SSRI. ${ }^{31}$ However, in our experience, patients with multiple symptoms who visit the psychosomatic unit are often oversensitive to side effects of drugs and tend to stop pharmacotherapy by selfdetermination at even minimal physical or psychiatric symptoms. Actually, 24 patients (15.4\%) refused to continue treatment based mainly on complaints of side effects within 2 weeks, even though the medicine was being taken at the minimum dose. In consideration of the side effects and adherence to the medication, the dose was limited to the intermediate dose in this study. Taking the converse point of view, the SSRI dose for patients with psychogenic somatoform symptoms, and not for patients with depression, may be able to be sparing, because symptoms improved in $83(62.9 \%)$ of 132 patients and $37(92.5 \%)$ of 40 patients with high $3 \mathrm{HB}$ (Table 1) under the sparing dose used in this study. However, the non-effective group could arguably respond to higher dosages of sertraline or venlafaxine.

Our study was also limited regarding the influence of anxiolytics, particularly benzodiazepines. Benzodiazepines are often used for insomnia and psychogenic somatoform symptoms in Japan, while SSRIs were the only first-line treatments preferred for cases with anxiety as the predominant symptom, possibly because only SSRIs are approved for anxiety disorders in Japan. ${ }^{32}$ Benzodiazepines were used by 44 patients $(33.3 \%)$ in this study. We compared the use of benzodiazepines regarding both serum $3 \mathrm{HB}$ levels and effectiveness of sertraline and venlafaxine, revealing no difference (data not shown). However, benzodiazepines are often clinically effective as the primary therapy for some patients with psychogenic somatoform symptoms, despite problems including dependence, withdrawal, decreased cognitive function, and increased risk of dementia and suicide. ${ }^{33-37}$ No studies have investigated whether SSRIs/SNRIs or benzodiazepines are appropriate for treating psychogenic somatoform symptoms, but levels of serum 3HB may provide useful guidance.

\section{Conclusion}

The present findings suggest that high serum $3 \mathrm{HB}$ in patients with psychogenic somatoform symptoms may be associated with suicidal ideation and the effectiveness of sertraline and venlafaxine with low-to-intermediate doses as SSRIs. The $3 \mathrm{HB}$ level may be a good predictor of the effectiveness of medication. Examination of serum $3 \mathrm{HB}$ levels may lead to earlier and more appropriate administrations of sertraline and venlafaxine.

\section{Disclosure}

The authors report no conflicts of interest in this work.

\section{References}

1. Katon WJ, Walker EA. Medically unexplained symptoms in primary care. J Clin Psychiatry. 1998;59:15-21.

2. Lepine J-P, Briley M. The epidemiology of pain in depression. Hum Psychopharmacol. 2004;19(S1):3-7. doi:10.1002/hup.618

3. Patetsos E, Horjales-Araujo E. Treating chronic pain with SSRIs: what do we know? Pain Res Manag. 2016;2016:2020915. doi:10.1155/ 2016/2020915

4. Grothe DR, Scheckner B, Albano D. Treatment of pain syndromes with venlafaxine. Pharmacotherapy. 2004;24(5):621-629. doi:10.1592/phco.24.6.621.34748 
5. Huang X, Li C, Luo YL, Wang B, Ji JL. Efficacy of venlafaxine extended-release monotherapy for first-episode depression with painful physical symptoms. Neuroreport. 2013;24(7):364-369. doi:10.1097/WNR.0b013e3283601a3e

6. Kaddurah-Daouk R, Krishnan KR. Metabolomics: a global biochemical approach to the study of central nervous system diseases. Neuropsychopharmacology. 2009;34(1):173-186. doi:10.1038/ npp.2008.174

7. Setoyama D, Kato TA, Hashimoto R, et al. Plasma metabolites predict severity of depression and suicidal ideation in psychiatric patients-A multicenter pilot analysis. PLoS One. 2016;11(12): e0165267. doi:10.1371/journal.pone.0165267

8. Sada N, Lee S, Katsu T, Otsuki T, Inoue T. Epilepsy treatment. Targeting LDH enzymes with a stiripentol analog to treat epilepsy. Science. 2015;347(6228):1362-1367. doi:10.1126/science.aaa1299

9. Youm YH, Nguyen KY, Grant RW, et al. The ketone metabolite beta-hydroxybutyrate blocks NLRP3 inflammasome-mediated inflammatory disease. Nat Med. 2015;21(3):263-269. doi:10.1038/ nm.3804

10. Sheehan DV, Lecrubier Y, Sheehan KH, et al. The mini-international neuropsychiatric Interview (M.I.N.I.): the development and validation of a structured diagnostic psychiatric interview for DSM-IV and ICD-10. J Clin Psychiatry. 1998;59:34-57.

11. Okun A, Stein RE, Bauman LJ, et al. Content validity of the psychiatric symptom index, CES-depression scale, and state-trait anxiety inventory from the perspective of DSM-IV. Psychol Rep. 1996;79 (3):1059-1069. doi:10.2466/pr0.1996.79.3.1059

12. Spielberger CD, Gorsuch RL, Lushene RE. Manual for the StateTrait Anxiety. Palo Alto, CA: Consulting Psychologist Press; 1970.

13. American Psychiatric Association. Diagnostic and Statistical Manual of Mental Disorders. 5th. (DSM-V). VA: American Psychiatric Publishing; 2013

14. 3-Hydroxybutyrate REAGENT KIT. KAINOS Co., Ltd. Tokyo, Japan. Available from: http://www.kainos.co.jp/jp/products/pdf/ TKA5520.pdf. Accessed April 23, 2021. (Japanese.)

15. Drug information sheet of sertraline. (English) Pfizer Pharmaceuticals Inc. Available from: https://www.rad-ar.or.jp/siori/english/kekka.cgi? $\mathrm{n}=1815$. Accessed April 23, 2021.

16. Drug information sheet of sertraline. (English) Pfizer Pharmaceuticals Inc. Available from: https://www.rad-ar.or.jp/siori/english/kekka.cgi? $\mathrm{n}=36222$. Accessed April 23, 2021.

17. Arora S, Henderson SO, Long T, et al. Diagnostic accuracy of point of care testing for diabetic ketoacidosis at emergency department triage: beta hydroxybutyrate versus the urine dipstick. Diabetes Care. 2011;34(4):852-854. doi:10.2337/dc10-1844

18. Noyes KJ, Crofton P, Bath LE, et al. Hydroxybutyrate near patient testing to evaluate a new end point for intravenous insulin therapy in the treatment of diabetic ketoacidosis in children. Pediatr Diabetes. 2007;8(3):150-156. doi:10.1111/j.1399-5448.2007.00240.x

19. Laffel LM, Wentzell K, Loughlin C, et al. Sick day management using blood 3-hydroxybutyrate (3-OHB) compared with urine ketone monitoring reduces hospital visits in young people with T1DM: a randomized clinical trial. Diabetes Med. 2006;23(3):278-284. doi:10.1111/j.1464-5491.2005.01771.x

20. Newman JC, Verdin E. Ketone bodies as signaling metabolites. Trends Endocrinol Metab. 2014;25(1):42-52. doi:10.1016/j. tem.2013.09.002

21. Cotter DG, Schugar RC, Crawford A. Ketone body metabolism and cardiovascular disease. Am J Physiol Heart Circ Physiol. 2013;304 (8):1060-1076. doi:10.1152/ajpheart.00646.2012
22. Chen L, Miao Z, Xu X. Beta-hydroxybutyrate alleviates depressive behaviors in mice possibly by increasing the histone3-lysine9-betahydroxybutyrylation. Biochem Biophys Res Commun. 2017;490 (2):117-122. doi:10.1016/j.bbrc.2017.05.184

23. Yamanashi T, Iwata M, Kamiya N, et al. Beta-hydroxybutyrate, an endogenic NLRP3 inflammasome inhibitor, attenuates stress-induced behavioral and inflammatory responses. Sci Rep. 2017;7(1):7677. doi:10.1038/s41598-017-08055-1

24. Kajitani N, Iwata M, Miura A, et al. Prefrontal cortex infusion of beta-hydroxybutyrate, an endogenous NLRP3 inflammasome inhibitor, produces antidepressant-like effects in a rodent model of depression. Neuropsychopharmacol Rep. 2020;40(2):157-165. doi:10.1002/npr2.12099

25. Manna V, Bolino F, Di Cicco L. Chronic tension-type headache, mood depression and serotonin: therapeutic effects of fluvoxamine and mianserine. Headache. 1994;34(1):44-49. doi:10.1111/j.15264610.1994.hed3401044.x

26. Keefe FJ, Shelby RA, Somers TJ, et al. Effects of copingskills training and sertraline in patients with non-cardiac chestpain: a randomized controlled study. Pain. 2011;152(4):730-741. doi:10.1016/j.pain.2010.08.040

27. Varia I, Logue E, O'Connor C, et al. Randomized trial of sertraline in patients with unexplained chest pain of noncardiac origin. Am Heart J. 2000;140(3):367-372. doi:10.1067/mhj.2000.108514

28. Aikens JE, Kroenke K, Nease DE, et al. Trajectories of improvement for six depression-related outcomes. Gen Hosp Psychiatry. 2008;30 (1):26-31. doi:10.1016/j.genhosppsych.2007.10.003

29. Hieronymus F, Nilsson S, Eriksson E. A mega-analysis of fixeddose trials reveals dose-dependency and a rapid onset of action for the antidepressant effect of three selective serotonin reuptake inhibitors. Transl Psychiatry. 2016;6(6):e834. doi:10.1038/tp.2016.104

30. Lisinski A, Hieronymus F, Eriksson E, et al. Low SSRI dosing in clinical practice-a register-based longitudinal study. Acta Psychiatr Scand. 2021;143(5):434-443. doi:10.1111/acps.13275

31. Redrobe JP, Bourin M, Colombel MC, et al. Dose-dependent noradrenergic and serotonergic properties of venlafaxine in animal models indicative of antidepressant activity. Psychopharmacology. 1998;138(1):1-8. doi:10.1007/s002130050638

32. Sakurai H, Uchida H, Kato M, et al. Pharmacological management of depression: Japanese expert consensus. J Affect Disord. 2020;266:626-632. doi:10.1016/j.jad.2020.01.149

33. Denis C, Fatséas M, Lavie E, et al. Pharmacological interventions for benzodiazepine mono-dependence management in outpatient settings. Cochrane Database Syst Rev. 2006;19:CD005194.

34. Lader M. Effectiveness of benzodiazepines: do they work or not? Expert Rev Neurother. 2008;8(8):1189-1191. doi:10.1586/ 14737175.8.8.1189

35. Lader M, Tylee A, Donoghue J. Withdrawing benzodiazepines in primary care. CNS Drugs. 2009;23(1):19-34. doi:10.2165/0023210200923010-00002

36. Penninkilampi R, Eslick GD. A systematic review and meta-analysis of the risk of dementia associated with benzodiazepine use, after controlling for protopathic bias. CNS Drugs. 2018;32(6):485-497. doi:10.1007/s40263-018-0535-3

37. Dodds TJ. Prescribed benzodiazepines and suicide risk: a review of the literature. Prim Care Companion CNS Disord. 2017;19(2). doi:10.4088/PCC.16r02037 


\section{Publish your work in this journal}

The International Journal of General Medicine is an international, peer-reviewed open-access journal that focuses on general and internal medicine, pathogenesis, epidemiology, diagnosis, monitoring and treatment protocols. The journal is characterized by the rapid reporting of reviews, original research and clinical studies across all disease areas. The manuscript management system is completely online and includes a very quick and fair peer-review system, which is all easy to use. Visit http://www.dovepress.com/ testimonials.php to read real quotes from published authors.

Submit your manuscript here: https://www.dovepress.com/international-journal-of-general-medicine-journal 